\title{
INVARIANT MEASURE, THE RECURRENCE THEOREM, AND THE ERGODIC THEOREM
}

\author{
FRED B. WRIGHT ${ }^{1}$
}

Let $(X, s, m)$ be a finite measure space, and let $T$ be a measurable transformation of $X$ into itself. The Individual Ergodic Theorem is said to hold for $T$ if for each measurable set $E$ in $X$, the averages

$$
\frac{1}{n} \sum_{j=0}^{n-1} f_{E}\left(T^{j} x\right)
$$

converge a.e. to a finite limit, where $f_{E}$ is the characteristic function of the set $E$. G. D. Birkhoff's Ergodic Theorem asserts this conclusion if $T$ is measure-preserving, in the sense that $m\left(T^{-1} E\right)=m(E)$ for each measurable set $E$. The same conclusion can be asserted under somewhat more general circumstances. We shall say that $T$ admits a finite, equivalent, invariant measure if there is a finite measure $p$ on $S$ such that $p \equiv m$ and such that $p\left(T^{-1} E\right)=p(E)$ for each measurable set $E$. If a measurable transformation $T$ admits such a measure, then the Individual Ergodic Theorem holds for $T$.

For any measurable transformation $T$, we say that the Recurrence Theorem holds for $T$ if, for any measurable set $E$ and any positive integer $n$, it is true that $m\left(E-\bigcup_{j=n}^{\infty} T^{-i} E\right)=0$. It is known [4] that a necessary and sufficient condition that the Recurrence Theorem hold is that $T$ be incompressible, in the sense that $E \subset T^{-1} E$ implies $m\left(T^{-1} E-E\right)=0$. A measure-preserving transformation on a finite measure space is incompressible, and consequently any transformation on a finite measure space which admits an equivalent, invariant, finite measure is also an incompressible transformation. These may be stated together.

THEOREM (PoINCARÉ-BIRKHOFF). If $T$ is a measurable transformation on a finite measure space $(X, \delta, m)$, and if $T$ admits a finite, equivalent, invariant measure, then both the Individual Ergodic Theorem and the Recurrence Theorem hold for $T$.

In a previous note [5], the following converse of this was established.

TheOREM. Let $T$ be a measurable transformation on a finite measure space $(X, \delta, m)$, for which both the Individual Ergodic Theorem and

Received by the editors September 16, 1959.

${ }^{1}$ Research Fellow of the Alfred P. Sloan Foundation. 
the Recurrence Theorem hold. Then $T$ admits a finite, equivalent, invariant measure.

In this note we shall be concerned with the continuous analogue of this result. Let $R_{+}$denote the right half-line of reals $0 \leqq t<\infty$. Suppose that for each $t \geqq 0$ there is given a measurable transformation $T_{t}$ of $X$ into itself, such that $T_{t} T_{u}=T_{t+u}$ for each $t, u \geqq 0$. Suppose also that $T_{0}$ is the identity transformation. Such a family is called a one-parameter semigroup of measurable transformations. If a oneparameter semigroup has the further property that $T_{t} x$ is jointly measurable in $t$ and $x$, we shall call the semigroup a measurable flow. If a measurable flow on a finite measure space $(X, s, m)$ has the property $\lim _{t \rightarrow u} m\left(T_{t}^{-1} E+T_{u}^{-1} E\right)=0$ for each measurable set $E$ and each $u \geqq 0$, we shall call the flow continuous. (Here, $A+B$ denotes the symmetric difference $(A-B) \cup(B-A)$.)

Let $\left\{T_{t}: t \geqq 0\right\}$ be a measurable flow in a finite measure space. We shall say that the flow admits a finite, equivalent, invariant measure if there exists a finite measure $p$ on $\mathcal{S}$ such that $p \equiv m$ and such that $p\left(T_{t}^{-1} E\right)=p(E)$ for any measurable set $E$ and any $t \geqq 0$. We note that if the flow admits such a measure, then each element $T_{t}$ in the flow is an absolutely continuous transformation of $X$, in the sense that if $m(E)=0$, then $m\left(T_{t}^{-1} E\right)=0$.

If $T_{t}$ is a continuous flow in a finite measure space, then for any $u \geqq 0$ and any measurable set $E$ in $X$, there is a measurable set $E^{u}$ having the properties: (i) $m\left(T_{t}^{-1} E-E^{u}\right)=0$ for any $t \geqq u$; (ii) if $F$ is any measurable set such that $m\left(T_{t}^{-1} E-E\right)=0$, for each $t \geqq u$, then $m\left(E^{u}-F\right)=0$. (This is a consequence of the completeness of the measure algebra of a finite measure space; see [3].) The continuity of the flow implies that such a set $E^{u}$ differs from the set $\cup\left\{T_{r}^{-1} E: r \geqq u, r\right.$ rational $\}$ by a set of measure zero. (See $[1$, p. 80$]$ or $[4, \S 3]$, for instance.) We will henceforth use the notation $E^{u}$, for any measurable set $E$ and any $u \geqq 0$, to denote $U\left\{T_{r}^{-1} E: r \geqq u, r\right.$ rational $\}$.

We say that the individual Ergodic Theorem holds for a measurable flow $\left\{T_{t}: t \geqq 0\right\}$ if the averages

$$
\frac{1}{v} \int_{0}^{v} f_{E}\left(T_{t} x\right) d t
$$

converge a.e. to a finite limit as $v \rightarrow \infty$, for each measurable set $E$, where $f_{E}$ is the characteristic function of $E$. We will denote this limit by $f_{E}^{*}(x)$. It is a consequence of Fubini's Theorem that for almost all $x \in X$, the integrals $\int_{0}^{o} f_{E}\left(T_{t} x\right) d t$ exist for all $v$.

For a continuous flow in a finite measure space, the Recurrence 
Theorem is said to hold if for each measurable set $E$ in $X$ and each $u \geqq 0$ it is true that $m\left(E-E^{u}\right)=0$. It is known [4] that a necessary and sufficient condition that the Recurrence Theorem hold is that the following conditions be satisfied simultaneously: $(\alpha)$ if $m\left(E^{0}-E\right)$ $=0$ then $m\left(E-E^{0}\right)=0 ;(\beta) m\left(E^{u}+E^{0}\right)=0$ for each measurable set $E$ and each $u \geqq 0$. The conditions are generalizations of the concept of incompressibility. It is easy to see that if a flow contains a single incompressible member, then $(\alpha)$ is satisfied. Since we have assumed that $T_{0}$ is the identity transformation, $(\alpha)$ is always satisfied. If every element $T_{t}$ in the flow is incompressible, then it is trivial to show that $(\beta)$ is satisfied. It is not known, however, if $(\beta)$ implies the incompressibility of each element in the flow.

The following theorem compresses both the Poincaré Recurrence Theorem and the Birkhoff Ergodic Theorem into a single statement.

Theorem. Let $\left\{T_{t}: t \geqq 0\right\}$ be a continuous flow on a finite measure space $(X, \delta, m)$. If the flow admits a finite, equivalent, invariant measure, then: (i) each $T_{t}$ is absolutely continuous; (ii) the Recurrence Theorem holds for the flow; and (iii) the Individual Ergodic Theorem holds for the flow.

We shall now establish the converse of this result.

Theorem. Let $\left\{T_{t}: t \geqq 0\right\}$ be a continuous flow in a finite measure space $(X, \mathcal{S}, m)$, such that (i) each $T_{t}$ is absolutely continuous, (ii) the Recurrence Theorem holds for the flow, and (iii) the Individual Ergodic Theorem holds for the flow. Then the flow admits a finite, equivalent, invariant measure.

Proof. By hypothesis

$$
\lim _{v \rightarrow \infty} \frac{1}{v} \int_{0}^{v} f_{E}\left(T_{t} x\right) d t=f_{E}^{*}(x)
$$

exists and is finite a.e. Then for any $u \geqq 0$, we have

$$
\lim _{n \rightarrow \infty} \frac{1}{n u} \int_{0}^{n u} f_{E}\left(T_{t} x\right) d t=f_{E}^{*}(x) \text { a.e. }
$$

Set $g_{E}(x)=u^{-1} \int_{0}^{u} f_{E}\left(T_{t} x\right) d t$; then this becomes

$$
\lim _{n \rightarrow \infty} \frac{1}{n} \sum_{j=0}^{n-1} g_{E}\left(T_{u}^{j} x\right)=f_{E}^{*}(x) \text { a.e. }
$$

If for each $x$ we set $K(x)=\left\{t \geqq 0: T_{t} x \in E\right\}$, then $f_{E}\left(T_{t} x\right)=f_{K(x)}(t)$. Because of the measurability of the flow, the set $K(x)$ is measurable. 
Moreover, $g_{E}(x)=(1 / u) \int_{0}^{u} f_{K(x)}(t) d t$, which is the relative measure of $K(x)$ in the interval $[0, u]$. Hence $0 \leqq g_{E}(x) \leqq 1$. By the Bounded Convergence Theorem of Lebesgue, $f_{E}^{*}$ is integrable, and

$$
\lim _{n \rightarrow \infty} \frac{1}{n} \sum_{j=0}^{n-1} \int_{X} g_{E}\left(T_{u}^{j} x\right) d m(x)=\int_{X} f_{E}^{*}(x) d m(x) .
$$

Since

$$
g_{E}\left(T_{u}^{j} x\right)=\frac{1}{u} \int_{j u}^{(j+1) u} f_{E}\left(T_{t} x\right) d t
$$

we have

$$
\begin{aligned}
\int_{X} g_{E}\left(T_{u}^{j} x\right) d m(x) & =\int_{X} \frac{1}{u} \int_{j u}^{(j+1) u} f_{E}\left(T_{t} x\right) d t d m(x) \\
& =\frac{1}{u} \int_{j u}^{(j+1) u} \int_{X} f_{E}\left(T_{t} x\right) d m(x) d t \\
& =\frac{1}{u} \int_{j u}^{(j+1) u} m\left(T_{t}^{-1} E\right) d t,
\end{aligned}
$$

by Fubini's Theorem. It follows that

$$
\int_{X} f_{E}^{*}(x) d m(x)=\lim _{n \rightarrow \infty} \frac{1}{u} \sum_{j=0}^{n-1} \frac{1}{n} \int_{j u}^{(j+1) u} m\left(T_{t}^{-1} E\right) d t
$$

for any $u>0$.

Now set $s_{0}(E)=u^{-1} \int_{0}^{u} m\left(T_{t}^{-1} E\right) d t$. Then $s_{0}(E) \geqq 0$, and clearly $s_{0}$ is finitely additive on $S$. If $\left\{E_{k}\right\}$ is any countable disjoint family of measurable sets, then we have $m\left(T_{t}^{-1} \cup_{k=1}^{\infty} E_{k}\right)=m\left(\cup_{k=1}^{\infty} T_{t}^{-1} E_{k}\right)$ $=\sum_{k=1}^{\infty} m\left(T_{t}^{-1} E_{k}\right)$ for each $t \geqq 0$. By the Monotone Convergence Theorem of Lebesgue, we have

$$
\int_{0}^{u} m\left(T_{t}^{-1} \bigcup_{k=1}^{\infty} E_{k}\right) d t=\sum_{k=1}^{\infty} \int_{0}^{u} m\left(T_{t}^{-1} E_{k}\right) d t .
$$

Therefore $s_{0}$ is countably additive. Since each $T_{t}$ is absolutely continuous, it follows that $s_{0} \ll m$. If we set $s_{j}(E)=s_{0}\left(T_{u}^{-j} E\right)$, then each $s_{j}$ is a measure with $s_{j} \ll m$, and we have

$$
\int_{X} f_{E}^{*}(x) d m(x)=\lim _{n \rightarrow \infty} \frac{1}{n} \sum_{j=0}^{n-1} s_{j}(E)
$$

for each measurable set $E$. It follows [2, p. 159] that if we set 


$$
p(E)=\int_{X} f_{E}^{*}(x) d m(x)
$$

then $p$ is a measure on $s$ and $p \ll m$. It is a consequence of (2) that the measure given by (1) is independent of $u$. From this fact and from (1) we have at once that $p\left(T_{u}^{-1} E\right)=p(E)$ for each $E$ and $u \geqq 0$. It also follows from (1) that if $E$ is an invariant set (that is, if $m\left(E+T_{u}^{-1} E\right)=0$ for each $\left.u\right)$, then $p(E)=m(E)$. In particular, $p$ is finite.

The Radon-Nikodym Theorem enables us to write $p(E)$ $=\int_{E} w(x) d m(x)$, for any measurable set $E$, where $w$ is a measurable function and where $w(x) \geqq 0$ a.e. $[m]$. To show that $p \equiv m$, it suffices to show that $w(x)>0$ a.e. $[m]$. Set $N=\{x \in X: w(x)=0\}$; then $p(N)=0$, and from the invariance of $p$ we have $p\left(T_{u}^{-1} N\right)=0$ for each $u \geqq 0$. Let $N^{0}$ be the set defined above; then $p\left(N^{0}\right)=0$. Since the Recurrence Theorem holds, we have $m\left(N^{0}+N^{u}\right)=0$ for any $u \geqq 0$. But clearly $m\left(N^{u}+T_{u}^{-1} N^{0}\right)=0$ for any $u \geqq 0$, and hence $m\left(N^{0}+T_{u}^{-1} N^{0}\right)$ $=0$. Therefore $N^{0}$ is an invariant set, and hence $m\left(N^{0}\right)=p\left(N^{0}\right)=0$. Since $N \subset N^{0}$, then we also have $m(N)=0$, and therefore $p \equiv m$. This completes the proof.

\section{REFERENCES}

1. Garrett Birkhoff, Lattice theory, Amer. Math. Soc. Colloquium Publications, vol. 25, New York, 1948.

2. N. Dunford and J. Schwartz, Linear operators. Part I, New York, Interscience, 1958.

3. I. E. Segal, Equivalences of measure spaces, Amer. J. Math. vol. 73 (1951) pp. 275-313.

4. F. B. Wright, Recurrence theorems and operators on Boolean algebras, to appear in Proc. London Math. Soc.

5. - The converse of the individual ergodic theorem, Proc. Amer. Math. Soc. vol. 11 (1960) pp. 415-420.

TUlane University 